\title{
A novel CT finding in cardiac amyloid
}

\author{
Authors: C Fielder Camm, ${ }^{\mathrm{A}, \mathrm{B}}$ Jaimal Kothari, ${ }^{\mathrm{C}}$ Victoria Stnoble ${ }^{\mathrm{D}}$ and Nikant $\mathrm{K}$ Sabharwal ${ }^{\mathrm{E}}$
}

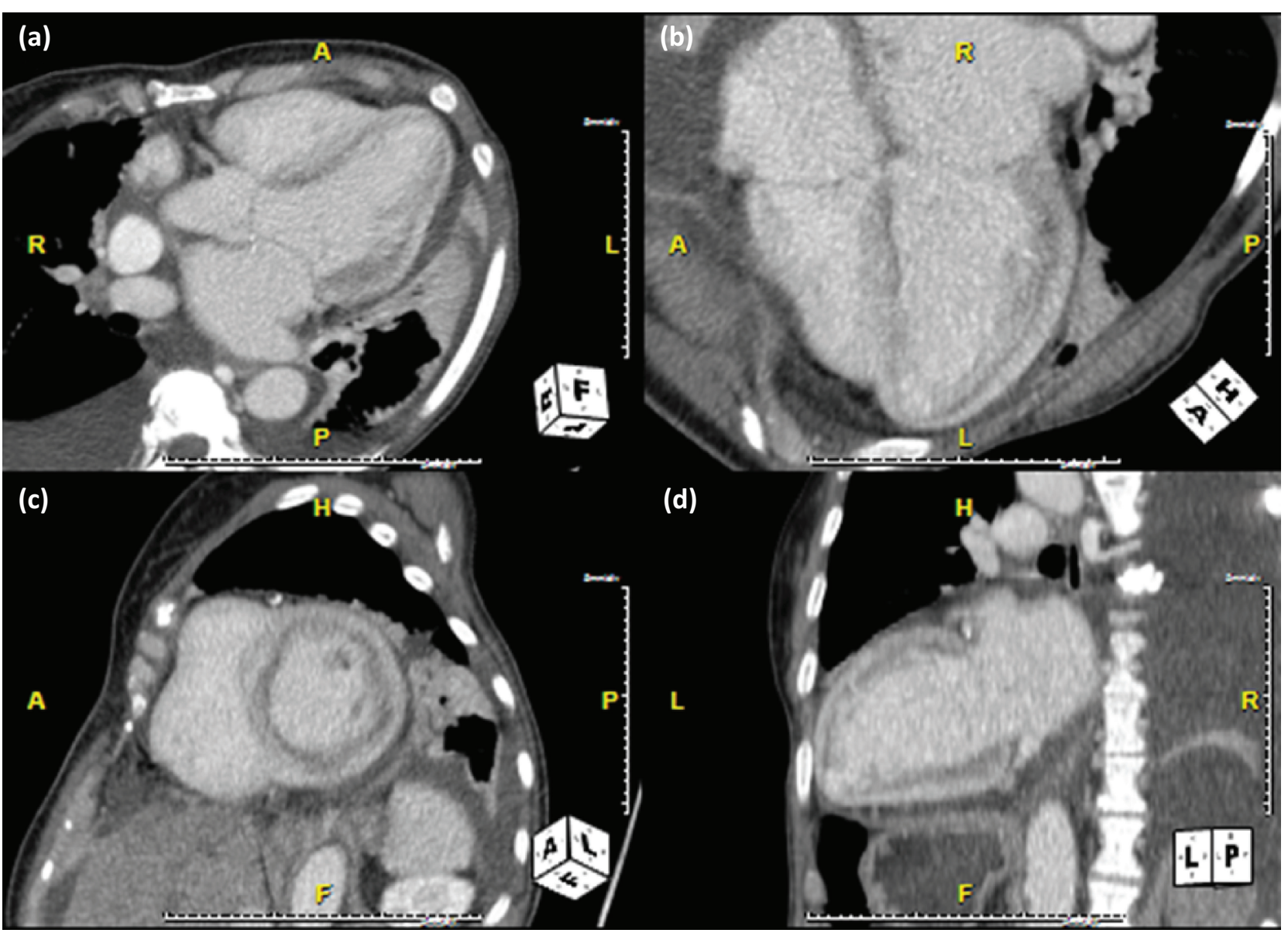

Fig 1. Cross-sectional contrast CT (computed tomography) image showing left ventricular sub-endocardial hypodensity. (a) Axial plane.

(b) 4-chamber view. (c) Short-axis view. (d) Long-axis view.

KEYWORDS: Amyloidosis, cardiac computed tomography

Authors: ${ }^{\text {A }}$ senior house officer, Department of Cardiology, Oxford University Hospitals NHS Foundation Trust, Oxford, UK; ${ }^{\mathrm{B}}$ academic clinical fellow, Radcliffe Department of Medicine, University of

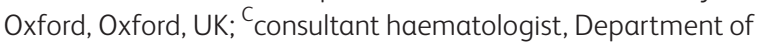
Haematology, Oxford University Hospitals NHS Foundation Trust, Oxford, UK; ${ }^{\mathrm{D}}$ consultant radiologist, Department of Radiology, Oxford University Hospitals NHS Foundation Trust, Oxford, UK; ${ }_{\text {E }}$ consultant cardiologist, Department of Cardiology, Oxford University Hospitals NHS Foundation Trust, Oxford, UK.
A 68-year-old woman presented with dyspnoea, having recently been diagnosed with multiple myeloma. She was reviewed in the cardiology clinic and an echocardiogram performed, revealing moderate concentric pseudo hypertrophy with sub endocardial signal hyperechogenicity consistent with infiltration. Although the left ventricular ejection fraction was normal, significant diastolic dysfunction was identified. All these echocardiographic findings 
are in keeping with cardiac amyloidosis. Brain natriuretic peptide level was $293.8 \mathrm{pg} / \mathrm{mL}$. Troponin I was raised at $0.2 \mu \mathrm{g} / \mathrm{L}$ (normal value $<0.04 \mu \mathrm{g} / \mathrm{L}$ ). A non-gated, contrast-enhanced venous-phase, computed tomography (CT) scan (undertaken as a myeloma staging scan) showed an extensive low density area in the sub endocardial layer of the left ventricle. This was associated with a small pericardial effusion and bilateral pleural effusions (Fig 1).

Given the clinical history and echocardiographic features, a diagnosis of cardiac amyloidosis was presumed, and urgent admission was arranged for inpatient chemotherapy. Examination revealed significant features of right-sided heart failure with a raised jugular venous pulse and bilateral pitting oedema to the knees. An electrocardiogram (ECG) on admission showed a ventricular tachycardia which was treated with amiodarone. On the second day of admission, the patient developed a junctional rhythm and then a progressively increasingly broad complex ventricular escape rhythm and a permanent pacemaker was inserted. Unfortunately, post pacemaker insertion she developed a pulseless electrical activity (PEA) cardiac arrest from which she could not be resuscitated. No pericardial effusion or pneumothorax was identified post pacemaker insertion.

Cardiac amyloidosis is associated with significant morbidity and mortality and is the most common cause of restrictive cardiomyopathy. ${ }^{1}$ Cardiac MRI and transthoracic echocardiography are the modalities of choice for evaluating cardiac amyloidosis. ${ }^{2}$ A characteristic pattern of diffuse heterogenous subendocardial increased signal is well described in cardiac MRI, ${ }^{3}$ and 'late enhancement' on gated cardiac CT has also been reported. ${ }^{4}$ However, this similar finding of low-density material in the subendocardial layer on a non-gated CT is a nove finding. Given the widespread use of CT assessment in patients being considered for transcatheter aortic valve insertion (TAVI), this feature may be useful in highlighting potential amyloidosis in this population.

\section{Acknowledgments}

The authors would like to acknowledge the assistance of Dr Niall Moore for his assistance in reporting the original CT scan.

\section{Consent to publish}

Consent to publish was obtained from the patient's next of kin.

\section{References}

1 Kushwaha SS, Fallon JT, Fuster V. Restrictive cardiomyopathy. N Engl J Med 1997;336:267-76.

2 Banypersad SM, Moon JC, Whelan C, Hawkins PN, Wechalekar AD. Updates in cardiac amyloidosis: a review. J Am Heart Assoc 2012;1:e000364.

3 Syed IS, Glockner JF, Feng D et al. Role of cardiac magnetic resonance imaging in the detection of cardiac amyloidosis. JACC Cardiovasc Imaging 2010;3:155-64.

4 Marwan M, Pflederer T, Ropers D et al. Cardiac amyloidosis imaged by dual-source computed tomography. J Cardiovasc Comput Tomogr 2008;2:403-5.

Address for correspondence: Dr NK Sabharwal, Department of Cardiology, John Radcliffe Hospital, Headley Way, Oxford OX3 9DU, UK.

Email: nikant.sabharwal@ouh.nhs.uk 